\title{
PROYECTO ALTAMIRA
}

\author{
(ALTAMIRA PROJECT) \\ Consorcio para Altamira. España
}

Fecha de recepción: 29-VIII-97

$142-159$

\section{RESUMEN}

En Cantabria, al N. de España, Altamira, enclave de gran importancia y concentración humana hace 100.000 años, nos abre las puertas de la prehistoria cuando María, en las excavaciones que realizaba su padre, D. Marcelino Sanz de Sautuola, en 1879, descubre las pinturas rupestres de lo que a partir de entonces será la "Cueva de Altamira", uno de los conjuntos rupestres más importantes del mundo.

La masiva afluencia de visitantes de las últimas décadas comienza a poner en peligro las pinturas, tomándose fuertes medidas restrictivas, que tan sólo permiten el acceso a 8.500 personas al año, desde 1982. Surge entonces la idea de la reproducción facsimilar de la cueva, englobada en el "Proyecto .Altamira", conjunto arquitectónico de primer orden encargado al arquitecto Navarro Baldeweg. En 1999, a las puertas de un nuevo milenio, Altamira podrá acoger de nuevo a cuantos visitantes quieran acercarse a la prehistoria.

\section{Introducción}

La Cueva de Altamira se encuentra enclavada en la cima de una colina, en terrenos de Vispieres, dominando un pequeño valle en el que, a unos $5 \mathrm{~km}$, se asienta la localidad de Santillana del Mar, importante conjunto de la época medieval, que dista unos $30 \mathrm{~km}$ de Santander. Dominan en el entorno prados y caseríos, respondiendo a una actividad agrícola-ganadera de tipo familiar.

Hace unos 100.000 años, otros moradores, los primeros que conocemos gracias a los útiles de piedra encontrados, se asentaron también allí al ofrecerles, sin duda, aquellas tierras, de gran belleza, cuanto podían necesitar.

Altamira debió ser un lugar de gran importancia y concentración humana por entonces. Caza, pesca, recursos forestales y fluviales preponderaron como medio de vida, plasmándose en sus manifestaciones artísticas, que convierten la Cueva en uno de los conjuntos de arte rupestre más importantes del mundo y de cuyo estudio ha surgido la formulación de diferentes teorías sobre el arte prehistórico.

Más tarde, durante el Paleolítico Superior, habitaron otros grupos humanos, en los períodos Solutrense y Magdaleniense, al que corresponden los "polícromos" del Gran Techo, datados en torno a los 13.000 años.

\section{La Cueva}

La Cueva, cavidad de desplome formada por los sucesivos hundimientos de la masa rocosa en fase de colapsamiento del Cárstico, alcanza los $270 \mathrm{~m}$ de longitud, encontrándose

(c) Consejo Superior de Investigaciones Científicas Licencia Creative Commons 3.0 España (by-nc)

\section{SUMMARY}

Up to the North of Spain, in Cantabria, Altamira, place of great importance a hundred thousand years ago, let us know how prehistoric days were when Mary, Marcelino Sanz de Sautuola's daughter, discovers in 1879, among the excavations her father was doing, the prehistoric paintings. Since then the Cave of Altamira became the paradigm of rupestrian art all over the world.

In the seventies, due to the excesive increase of visitors that imperil the paintings, strong restricted measures were taken. Since 1982, only 8.500 people per year are allowed to visit the Cave. Then, the idea of an Altamira's reproduction was borned. The "Altamira Project", a very important architectural unit being managed and promoted by a partnership created on that purpose is ordered to the renowned architect Navarro Baldeweg. In 1999, being at a new millennium's door, Altamira will receive again all the visitors looking for prehistoric times.

la Sala de Pinturas a una media de $7 \mathrm{~m}$ de profundidad, apreciándose en su interior una serie de abultamientos y grietas sobre los que fueron realizadas las pinturas, intentando, al aprovechar estos volúmenes, dotarlas de movimiento, consiguiendo, así, el mayor realismo posible.

Si bien el yacimiento paleolítico ya era conocido años antes, fue, a raíz de las excavaciones llevadas a cabo en 1879 por D. Marcelino Sanz de Sautuola en la cueva que Modesto Cubillas descubre inicialmente, poniéndolo en su conocimiento, cuando su hija, María, sin saberlo entonces, pasa a la historia al encontrar las pinturas.

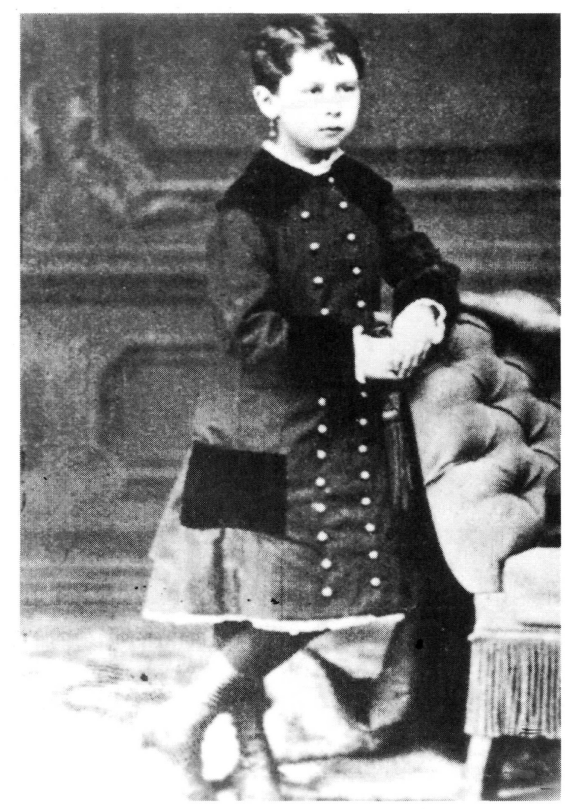

Maria Sanz de Sautuola. 
A partir de entonces, el Ayuntamiento de Santillana del Mar toma medidas para garantizar la conservación de la cueva que ya se vislumbra como algo único, especial y sumamente frágil, si bien no será hasta 1901, a raiz del descubrimiento de los grabados de Les Combarelles y las pinturas de Font-de-Gaume en Francia, cuando se marcará el inicio del reconocimiento de Altamira.

En 1924, queda declarada Monumento Arquitectónico Artístico y, en el año 1933, una vez refundidas todas las clasificaciones existentes, Monumento Histórico-Artístico, iniciándose una considerable afluencia de visitantes en la década de los 40 .

Simultáneamente, Lascaux, descubierta ese mismo año y masivamente visitada a partir de entonces, debe cerrarse al público, finalmente, en 1963, ante el alarmante deterioro de las pinturas, naciendo, en ese momento, la idea de realizar una réplica, cuyos estudios se prolongaron hasta los años 80 .

Altamira sigue los mismos pasos. La gráfica estadística del número total de visitantes anuales crece incesante, pasando de unos 30.000 en 1952 a 60.000 en $1957,155.000$ en 1967 y 175.000 en 1973, determinándose, tras una serie de estudios e informes competentes, su cierre definitivo en 1977. A los 5 años, en 1982, con enormes condiciones restrictivas, se reabre, permitiéndose el acceso únicamente a 8.500 personas al año. Nace entonces, al igual que sucediera en Lascaux, la idea de la Réplica de la Cueva, ante la demanda masiva de público que, paciente, en interminables listas de espera, aguarda su turno para entrar en la Prehistoria.

Después de 15 años, estando Altamira, desde 1985, incluida en la lista del Patrimonio Mundial por la UNESCO y, tras numerosos convenios y acuerdos, se crea el Consor- ciopara Altamira, integrado por el Ministerio de Educación y Cultura, la Diputación Regional de Cantabria, el Ayuntamiento de Santillana del Mar y la Fundación Marcelino Botín, para gestionar el proyecto del Museo Nacional y Centro de Investigación de Altamira, que contenga en su interior la reproducción facsimilar de la cueva, encargando su redacción al Dr. Arquitecto Juan Navarro Baldeweg y potenciando, además, el tan necesario desarrollo turístico, cultural y económico de toda la región.

\section{EI Proyecto}

\subsection{Diseño}

La construcción de la Réplica formará un conjunto arquitectónico en el que se integrarán un Centro de Investigación, un museo, una nueva biblioteca y un almacén de fondos arqueológicos, que responderán a las necesidades de difusión y expectativas de afluencia de público. La capacidad de estos espacios expositivos y de almacenamiento será tal que podrán acoger, en depósito, los fondos de Prehistoria/Paleolítico del Museo Regional de Prehistoria y Arqueología de Cantabria y su crecimiento durante los próximos años.

El nuevo edificio, con una superficie construida de unos $6.748 \mathrm{~m}^{2}$, se situará próximo a la Cueva, más allá de una zona de abundante vegetación, junto a su costado occidental, garantizando la conservación de su entorno original, al rodearla de un paisaje liberado y limpio que conserve la capacidad evocativa que el misterio de su entidad exige. Por ello, la afluencia masiva de visitantes quedará debidamente ordenada y organizada, procurándose el mínimo deterioro posible en todos los órdenes.

En líneas generales, el Museo, la Réplica y el Centro de

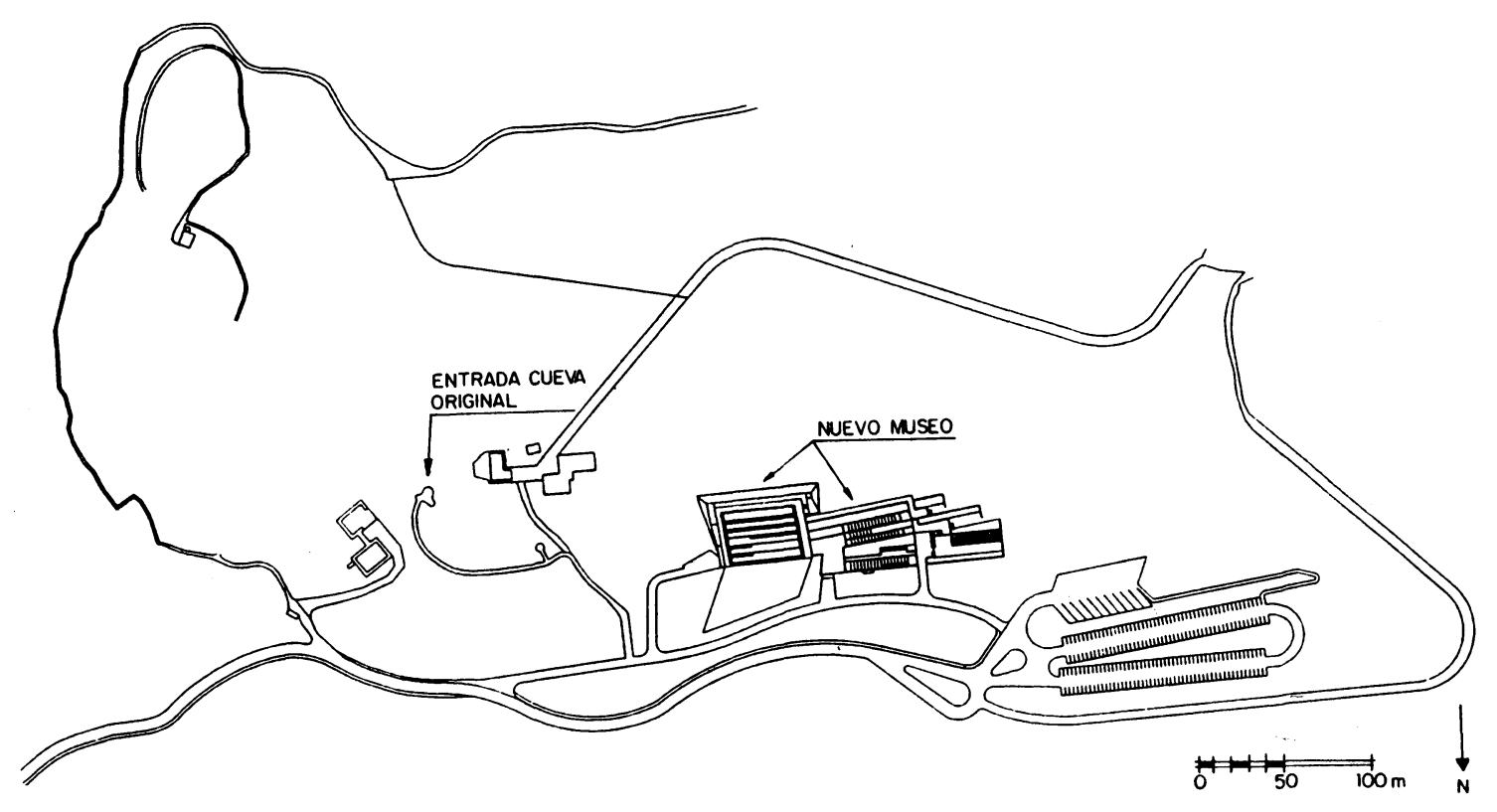

Planta de situación. 


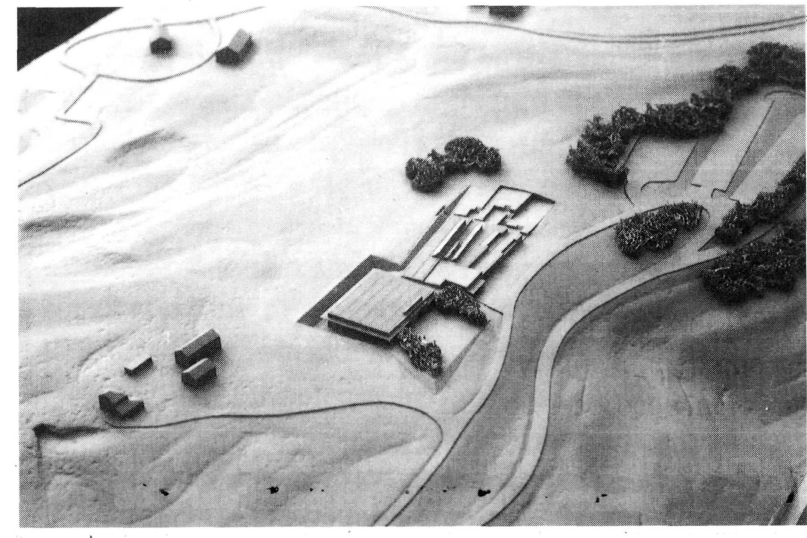

Maqueta de la situación del nuevo Museo.

Investigación proyectados, se dividen en dos áreas de edificación diferenciadas: por un lado, la zona en que se aloja la Réplica y, por otro, unas formas que se extienden linealmente desde el vestíbulo principal, que dan acogida a los visitantes. Los tres brazos de este área contendrán los espacios de exposición permanente y temporal, las salas de usos múltiples, la sala de actos, venta de publicaciones y un restaurante o cafetería, que se prolonga en su uso al aire libre.

La estructura primaria de estas formas, desplegadas casi en paralelo, no responde a una única tipología estructural, integrando en su definición una serie de sistemas estructurales diferenciados, adecuados a las necesidades resistentes, funcionales y constructivas de las diferentes zonas del edificio.

El esquema fundamental de la estructura resistente consiste en un sistema de hormigón armado en el que se integran también distintos elementos de carácter mixto de hormigón y acero estructural, para poder solventar adecuadamente los puntos más solicitados del conjunto y favorecer, sobre todo, la construcción de la obra.
En líneas generales, los soportes están constituidos por piezas rectangulares de hormigón armado, salvo en el caso de la planta baja, situada encima de la Réplica de la Cueva, en la que la estructura portante está formada por una estructura metálica, un forjado mixto colaborante de chapa plegada y hormigón, suspendida por medio de una serie de tirantes metálicos, de acero, anclados a los fondos de las grandes vigas mixtas portantes de la cubierta exterior.

La cubierta, elemento sustancial del proyecto, dadas las grandes dimensiones que la cobertura de la Réplica requiere, añade a su misión específica otra, más importante aún, si cabe: conseguir la máxima integración del edificio en su entorno, proponiendo el proyecto, para ello, una cubierta inclinada, siguiendo las pendientes del suelo natural y sembrada de césped.

Se divide en dos grandes zonas, bien diferenciadas: la zona de la Réplica de la Cueva y la zona del vestíbulo de entrada, salas de exposiciones, salón de actos, aulas-taller, venta de publicaciones y cafetería.

En esta primera zona y debido, principalmente, a las grandes luces, 41,5 metros, a la existencia de grandes lucernarios corridos que separan o subdividen esta parte del edificio y a las apreciables cargas de las vigas, tanto superiores como colgadas de las mismas, los esfuerzos en las vigas-puente que cubren la sala son de una importancia considerable. Para lograr una respuesta adecuada entre las relaciones de resistencia, coste, facilidad de ejecución y conservación, se ha considerado, como solución más favorable, el empleo de grandes piezas mixtas pretensadas de hormigón y acero estructural.

\subsection{Estructura}

La estructura de esta parte de la cubierta se completa con la existencia de dos grandes marquesinas laterales de 3 y

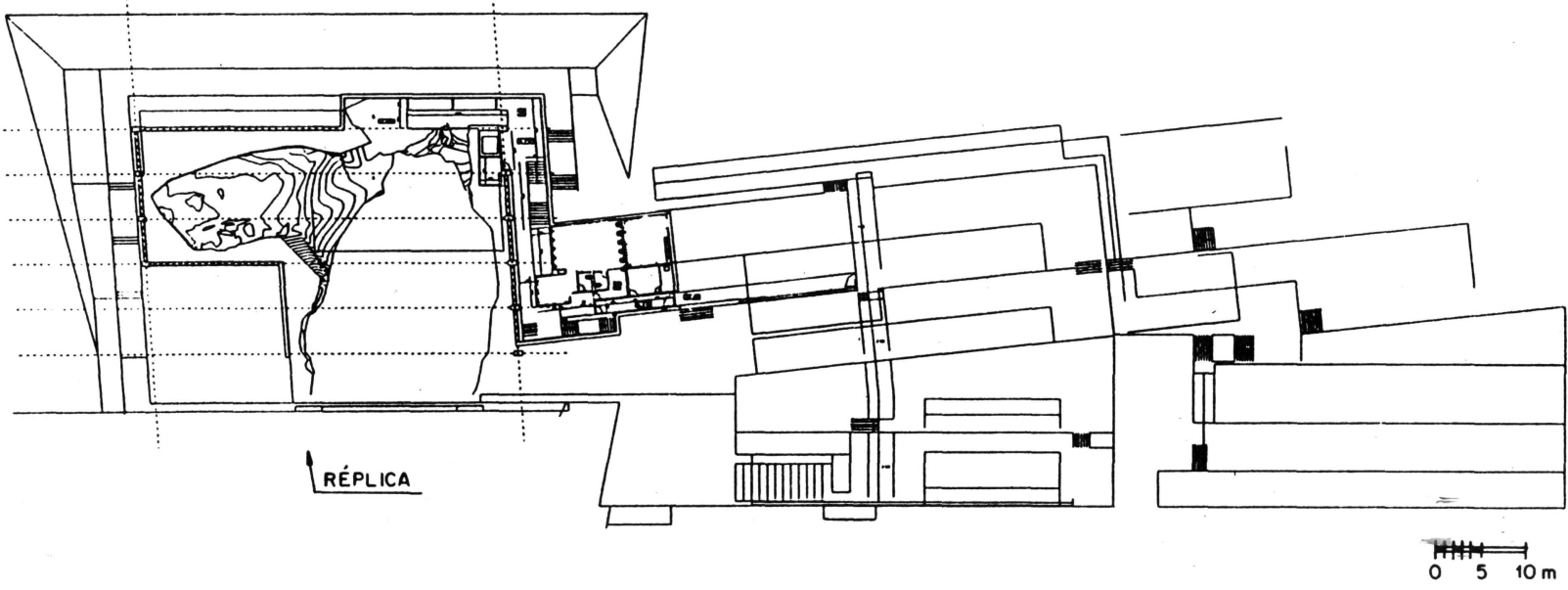


4 metros de luz, en voladizo. que constituyen parte importante de la imagen del edificio y que se realizan en hormigón armado, directamente unidas a las hileras de soportes de cada lado de la sala, mediante dobles piezas de vigas separadas entre sí, recibiendo, una de ellas, reacciones verticales hacia abajo y la otra hacia arriba. En ambos casos se disponen las adecuadas contraflechas de ejecución. En las otras dos marquesinas, frontal y dorsal, que se unen de forma directa con las grandes vigas de borde, se ha preferido emplear elementos ligeros metálicos, separados cada metro, que se recubrirán con materiales ligeros, para evitar difíciles uniones y esfuerzos elevados de las mismas.

En la segunda zona, debido a su configuración escalonada y fragmentada, es obligado establecer diversas disposiciones resistentes, unas veces longitudinales y otras transversales, apoyadas en nervios de mayor o menor entidad, proponiéndose una cubierta con losas de hormigón armado unidireccionales apoyadas en vigas tipo, también de hormigón armado o en grandes vigas prefabricadas en hormigón pretensado.

En los lucernarios de esta parte del edificio aparecen una serie de piezas trianguladas de grandes dimensiones que, a la vez que dan forma a los lucernarios, cubiertas y faldones, constituyen grandes vigas cajón de sección irregular que se apoyan longitudinalmente en el borde opuesto al lucernario, sobre potentes piezas prefabricadas en hormigón pretensado y, en sus extremos, en sendos diafragmas de hormigón armado que, a su vez, reposan sobre los soportes o sobre las mismas vigas pretensadas de cubierta citadas.

La estructura de planta baja presenta, igualmente, zonas muy diferenciadas que han requerido tratamientos específicos

Sobre la Réplica de la Cueva, la necesidad de dejar ésta completamente libre de soportes, obliga a que las superficies de planta baja situadas sobre la misma deban ir suspendidas de la cubierta. Por esta razón, para aligerar al máximo el peso de tales zonas y favorecer su proceso constructivo, se emplea una estructura metálica suspendida de tirantes, también metálicos, que se completa con un forjado en losa mixta de chapa plegada y capa de compresión de hormigón.

En la zona del vestíbulo de entrada, salas de exposiciones, salón de actos, aulas-taller, venta de publicaciones y cafetería, la estructura de la planta y todos los casos de vigas, muros y muretes de hormigón armado, en los que se apoyan forjados tradicionales, se han considerado, en proyecto, formados por placas pretensadas alveoladas autoportantes, de modo que, al estar muy cerca del terreno subyacente, no necesiten ser apeadas y su ejecución sea lo más rápida y eficaz posible.
En la planta sótano, toda ella sobre solera, destacan como único elemento estructural, los muros de contención periféricos y, en la zona de la Réplica, el sistema de muros, de gran porte, que circunda el espacio necesario, haciendo frente a los empujes del terreno.

Para el diseño y cálculo de las cimentaciones, debido a la presencia muy somera de la calcarenita, roca que forma el estrato competente para las cimentaciones, se han empleado soluciones de tipo directo: zapatas.

A pesar de la complejidad estructural, su ejecución puede llevarse a cabo de modo convencional, siguiendo los procesos habituales en estructuras de hormigón armado para edificación, si bien existen algunos elementos y zonas en las que se deben seguir unas condiciones y pautas determinadas.

\subsection{Instalaciones}

El planteamiento de las instalaciones, fundamental en el funcionamiento del complejo, está dotado para responder a los requerimientos normativos y funcionales, garantizando las condiciones de comodidad y las necesidades en materia de seguridad, protección contra incendios, comunicación y transporte.

En su diseño se han tenido en cuenta, en primer lugar, las particulares características del entorno, con objeto de no alterar el equilibrio medioambiental del área de las cuevas, asegurando, a la vez, la eficiencia energética del conjunto, la máxima discreción acústica y visual de los equipos en espacios interioresy exteriores, flexibilidad en su utilización y total fiabilidad del equipo y materiales, asegurando la protección activa y pasiva del edificio, usuarios y visitantes.

Para dar solución a todos estos requerimientos, el proyecto propone la creación de un túnel de conducciones bajo la cota de planta inferior, que asegure la accesibilidad a los locales de instalaciones con el mínimo de interferencias.

Las salas de máquinas se sitúan en zonas que aseguran un mínimo impacto visual y acústico, se programa la utilización de sistemas de tratamiento unitario por zona de uso diferenciado, se utiliza energía gratuita de condensación para el control de humedad de los locales, se instalan equipos de alumbrado de bajo consumo, con una zonificación eléctrica acorde con el uso previsible de los locales y. por último, se proyecta un sistema de gestión central de instalaciones con programas de ahorro energético, usos, horarios y mantenimiento preventivo.

El proyecto trata en todo punto de reducir el impacto negativo de las construcciones, necesarias para albergar un programa de tan extensa superficie. La arquitectura, no obstante, tiene un valor propio y ello se hace evidente en formas que emergen según una geometria flexible, 

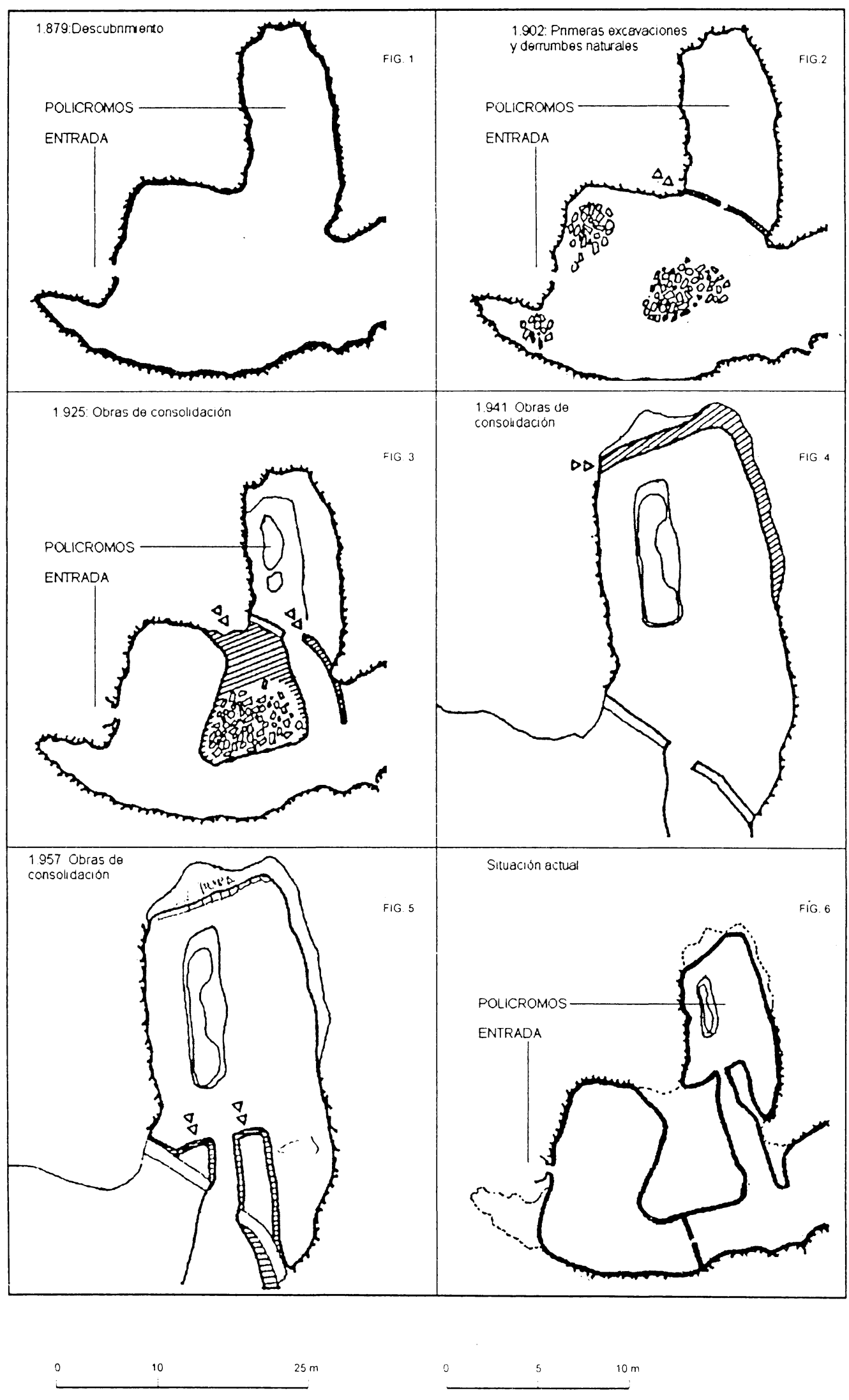

FIGURAS $1.2 .3 \times 6$

FIGURAS 4 YS

Transformaciones en el vestibulo y sala de pinturas. 
adaptándose a la ladera, creando una topografia artificial que se combina con las cubiertas plegadas que sobresalen con un carácter minimalista, originándose, gracias a un sistema de lucernarios corridos, unos espacios interiores en los que la luz natural y artificial, entrelazadas, son protagonistas.

La creación de la Réplica invita a interpretar su instalación en el paisaje como "imagen espejo" o reflejada, que supone un ámbito "virtual". Por ello, el marco que establece la diferencia entre lo virtual y lo real cobra importancia, manifestándose como un perímetro recortado en la colina, subrayado por marquesinas en voladizo de tres y cuatro metros, albergando la Réplica en su interior.

Desde dentro, enmarcado en el contorno de la boca de entrada, se recorta el paisaje lejano, mientras que hacia el interior, donde materiales tan nobles como la piedra y madera alternan su perfecta cadencia, las condiciones lumínicas de la Cueva producen un efecto de luz rasante, similar al de la cueva original, avivando, como si se tratase de una naturaleza invariable en el tiempo, el relieve de la bóveda y favoreciendo la interpretación correcta de las formas que inspiraron las pinturas reforzandosu animación, de modo que, al ser eliminadas, además, las diversas transformaciones que obras de refuerzo y consolidación han producido en paredes y techos; los muros que han aislado las pinturas polícromas del resto de la Cueva y su entrada, colapsada hace unos 14.000 años, los nuevos visitantes del año 2000 podrán contemplarla como sus creadores la conocieron, habitaron y dibujaron.

En suma, el proyecto de Navarro Baldeweg, con más de $3.000 \mathrm{~m}^{2}$ construidos y 1.700 para terrazas y áreas de picnic, cuya terminación en 20 meses está prevista para 1999 y cuyas obras, por valor de 1.179 millones de pesetas han sido adjudicadas a NECSO, estimándose un coste final del conjunto -edificios, Réplica, accesos, recuperación paisajística del entorno y dotación interiorde unos 2.400 millones de pesetas, se inicia en el mes de octubre, combinando los alardes constructivos que una actuación de estas características requiere en los albores del siglo XXI con la máxima integración en el paisaje, utilizando materiales y tecnología punta para lograrlo. Así, cubierta jardín, lucernarios corridos, lamas de aluminio, sillería y mampostería de piedra natural y dorada, que soportan una estructura de paneles de aluminio lacados, en color ocre, componen una imagen fragmentada y escalonada, distinguiéndose dos planos fundamentales: el soporte que emerge del suelo y la cubierta soportada como lámina recortada, flotando en el relieve del suelo natural.

Se abordarátambién una reestructuración medioambiental, para que no sólo la Cueva sino cuanto la circunda, recreado lo más fielmente posible, pueda hacernos revivir, como si de un viaje al pasado se tratase, la vida del hombre de Altamira, la Prehistoria.

Ficha Técnica

Entidad promotora: Consorcio para Altamira

Arquitecto: Juan Navarro Baldeweg

Constructora: NECSO 